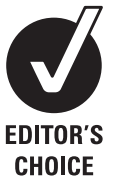

'Department of Ophthalmology, Centre for Eye Research Australia, University of Melbourne, Australia ${ }^{2}$ Department of Epidemiology, Shenzhen University School of Medicine, Shenzhen, Guangdong, PR China ${ }^{3}$ Department of Evidence-Based Medicine, Cardiovascular Institute and Fu Wai Hospital, Chinese Academy of Medical Sciences and Peking Union Medical College, Beijing, PR China ${ }^{4}$ Tulane University School of Public Health and Tropical Medicine, New Orleans, Louisiana, USA

\section{Correspondence to}

Dr Dongsheng Hu, Department of Epidemiology, Shenzhen University School of Medicine, 3688Nanhai Avenue, Shenzhen, Guangdong 518060, PR China; dongsheng_hu@hotmail.com

Accepted 7 May 2009

\title{
A quick self-assessment tool to identify individuals at high risk of type 2 diabetes in the Chinese general population
}

\author{
Jing Xie, ${ }^{1}$ Dongsheng $\mathrm{Hu}^{2}{ }^{2}$ Dahai $\mathrm{Yu}^{3}$ Chung-Shiuan Chen, ${ }^{4}$ Jiang $\mathrm{He}^{4}{ }^{4}$ Dongfeng $\mathrm{Gu}^{3}$
}

\section{ABSTRACT}

Background Currently available tools for identifying individuals at high risk of type 2 diabetes can be invasive, costly and time consuming. This study aims to develop and validate a self-assessment tool for identifying individuals at high risk of type 2 diabetes in the Chinese general population.

Methods A cross-sectional survey was conducted from 2000 to 2001 in a nationally representative sample of 15540 Chinese adults aged $35-74$ years. The diabetes risk level (DRL) was assessed by classification and regression tree (CART) analysis using four predictors: age, body mass index, waist-hip ratio (WHR) and waist circumference (WC).

Results The significant predictors for type 2 diabetes were WHR and age for women and WC and age for men. The categories generated by CART analysis stratified women into eight DRL and men into five DRL. The prevalence of type 2 diabetes increased with the increase in DRL in both women and men. $A D R L$ of 6 or greater predicted type 2 diabetes status with a sensitivity of 0.61 (95\% $\mathrm{Cl} 0.55$ to 0.67$)$, a specificity of 0.71 (95\% Cl 0.70 to 0.73 ) in women, and a DRL of 3 or greater predicted type 2 diabetes status with a sensitivity of $0.59(95 \% \mathrm{Cl} 0.52$ to 0.65$)$ and a specificity of $0.63(95 \% \mathrm{Cl} 0.62$ to 0.65$)$ in men. Conclusions This study demonstrates that application of the DRL has identified a substantial proportion of individuals with type 2 diabetes in the Chinese general population. It suggests that there is a great potential for applying the self-assessment tool in healthcare-limited settings.

The rising prevalence of type 2 diabetes has a become major public health problem worldwide, and it has been predicted to affect 366 million people by the year 2030, of which at least 40 million people would be from China alone. ${ }^{1}$ Approximately a $1 \%$ increase in the prevalence of type 2 diabetes will result in one million more patients in China, which imposes a massive socialeconomic burden on the community.

Studies have reported that type 2 diabetes is associated with a number of genetic, environmental and lifestyle factors. ${ }^{2-7}$ Recent intervention studies have also clearly shown that type 2 diabetes could be prevented or delayed through simple lifestyle modification among high-risk individuals. ${ }^{8-10}$ Therefore, the earlier detection of individuals at high risk of type 2 diabetes is a high priority for primary prevention. However, currently available screening tools for identifying individuals at high risk of type 2 diabetes can be invasive, costly and time consuming. ${ }^{11-18}$ Furthermore, most diabetes risk scores have been developed in western populations, ${ }^{11} 13-15171920$ south Asian ${ }^{12} 182122$ and Arab populations ${ }^{23}$ with application in clinical practice/ primary care services. ${ }^{15}$ 17-19 Such predicting tools are lacking in the Chinese population. We also note that different risk scores are not extrapolated across populations. ${ }^{20}$ In addition, some of these risk scores used laboratory tests and required a history of medication, ${ }^{12} 13151618$ which limited their use in China due to limited healthcare resources, especially in private-financing healthcare services. ${ }^{24}$ Easy selfassessment anthropometric measures have been suggested to be good indictors for type 2 diabetes in different populations, ${ }^{25}$ and might be used as alternatives for developing screening tools.

So far, no screening tool for type 2 diabetes has been reported in the Chinese population, which results in a large proportion of undiagnosed type 2 diabetes in China. ${ }^{26}$ In the current study, we aim to develop and validate a self-assessment tool for identifying individuals at high risk of type 2 diabetes in the Chinese general population.

\section{RESEARCH DESIGN AND METHODS \\ Study population}

Our analyses are based on data collected from the International Collaborative Study of Cardiovascular Disease in Asia (the InterASIA) conducted in China. The method of this study has been described in detail elsewhere. $^{25-28}$ In brief, a four-stage stratified sampling method was used to select a nationally representative sample of the general population in China from 2000 to 2001. A total of 19012 persons was randomly selected from 20 primary sampling units (street districts in urban areas or townships in rural areas). Of the 19012 persons, 15838 participants (15540 participants aged 35-74 years) completed the survey and examination (response rate 83\%). The current study included 994 persons with type 2 diabetes and 13129 persons with normal fasting glucose aged 35-74 years. Participants with impaired fasting glucose $(n=1121)$ and those with missing fasting glucose measurements $(n=296)$ were excluded from this analysis.

The InterASIA study was approved by the Institutional Review Board at the Tulane University Health Sciences Center and the ethics committee and other relevant regulatory bodies in China. Informed consent was obtained from each participant.

\section{Data collection}

Data collection was conducted in examination centres at local health stations or community clinics by trained research staff using a standard 
questionnaire. Information included age, gender, self-reported history of diabetes and the previous diagnosis and treatment of diabetes.

\section{Anthropometric measurements}

Body weight, height, waist circumference (WC) and hip circumference were measured by trained observers according to a standard protocol in the InterASIA. ${ }^{26}$ Body mass index (BMI) was calculated as weight (in kilograms) divided by height (in metres) squared. Waist-hip ratio (WHR) was calculated as WC divided by hip circumference.

\section{Laboratory measurements}

Blood specimen samples were obtained to determine plasma glucose after at least $12 \mathrm{~h}$ of fasting and were collected by the use of vacuum tubes containing sodium fluoride. The serum glucose level was measured by using the modified hexokinase enzymatic method (Hitachi automatic clinical analyser, model 7060, Japan).

Participants without a previous diagnosis of diabetes were categorised according to the American Diabetes Association diagnostic criteria as follows: undiagnosed diabetes (fasting glucose $\geq 7.0 \mathrm{mmol} / \mathrm{l})$ and impaired fasting glucose $(6.1-6.9 \mathrm{mmol} / \mathrm{l}) .{ }^{29}$ Diabetes was defined as a self-reported history of diabetes plus undiagnosed diabetes. Obesity was defined as a BMI of $28 \mathrm{~kg} / \mathrm{m}^{2}$ or greater, overweight was defined as a BMI of 24 or greater but less than $28 .^{30}$

\section{Data analyses}

Analysis was conducted in women and men separately. Before building models, the dataset was randomly split into two subset samples, $50.2 \%(n=7083)$ of the data for the training sample and the remaining $49.8 \%(n=7040)$ for the testing sample. Continuous variables were expressed as median (25th percentile, 75 th percentile) and categorical variables as $\mathrm{n}(\%)$. The $\chi^{2}$ test was used to analyse categorical data. Differences in continuous variables were evaluated by the Mann-Whitney Wilcoxon test. Classification and regression tree (CART) analysis was used to develop a simple self-assessment tool for identifying individuals at high risk of type 2 diabetes. This procedure repeatedly partitioned the data to create subgroups with highly homogenous outcomes. ${ }^{31}$ CART constructs a tree that will separate the data in the 'best' way by finding binary splits on variables; finds the best splitting variable and the best splitting point at each stage. The decision rule was determined by the Gini criterion, a measure of variability within the new subgroups. ${ }^{32}$ The target variable has a value of 0 or 1 depending on diabetes status ( 0 if absent, or 1 if present). The explanatory variables selected to predict the risks of type 2 diabetes consist of four easily attainable selfassessment continuous variables (age, BMI, WC and WHR). Nodes in the CART analysis were constrained to have a minimum size of 400 records in parent nodes and 200 records in final child nodes. The proportion of subjects having type 2 diabetes was reported in each node of the tree. A risk stratification system (diabetes risk level; DRL) was developed based on the final child nodes in CART analysis.

A multivariable logistic regression model including the same predictors was also developed. The predictive performance of CART analysis was evaluated by using the area under the receiver operating characteristics curve (AUC), sensitivity, specificity, the positive predictive value (PPV) and the negative predictive value (NPV).

All analyses were performed using STATA version 9.2 except the CART analysis was conducted using the SPSS 16.0 statistical software package. A $p$ value less than 0.05 was considered statistically significant.

\section{RESULTS}

Comparison of the characteristics of the 7083 participants used to develop the model (training sample) and the 7040 participants used to test the model (testing sample) are shown in table 1 . The training sample and the testing sample were similar with respect to age, WHR, BMI, WC and the prevalence of type 2 diabetes. The overall prevalence of type 2 diabetes was $6.4 \%$ (95\% CI $5.8 \%$ to $6.9 \%)$ and $6.7 \%(95 \%$ CI $6.1 \%$ to $7.3 \%)$ for women and men, respectively.

Figures 1 and 2 depict the classification tree along with the prevalence of type 2 diabetes in each child node. Of the four common easily known predictors (age, WHR, WC and BMI), WHR and age were selected by the CART analysis for women, whereas WC and age were selected for men. WHR was proved to be the best predictor for women $\left(\chi^{2}=149.6, p<0.001\right)$ and WC for men $\left(\chi^{2}=64.01, p<0.001\right)$. Age was the second most important predictor for type 2 diabetes in both women and men, respectively.

The final child nodes generated by CART analysis stratified women into eight DRL and men into five DRL (figures 1 and 2). The prevalence of type 2 diabetes increased with the increase in DRL in both women and men, as shown in figure 3 (women $\chi_{\text {trend }}^{2}=2059.42, \quad \mathrm{p}<0.001 ;$ men $\left.\chi_{\text {trend }}^{2}=619.01, \quad \mathrm{p}<0.001\right)$. Women with a DRL of 8 (WHR $>0.918$ ) and men with a DRL of 5 (WC $>84 \mathrm{~cm}$ ) are at the highest risk of type 2 diabetes.

Sensitivity, specificity, PPV, NPV and likelihood ratios that predict the presence of type 2 diabetes for a threshold are shown

Table 1 Sociodemographic and clinical characteristics of study participants in the training sample and the testing sample, by gender

\begin{tabular}{|c|c|c|c|c|c|c|}
\hline \multirow[b]{2}{*}{ Characteristics } & \multicolumn{3}{|l|}{ Women } & \multicolumn{3}{|l|}{ Men } \\
\hline & Training sample & Testing sample & $\overline{p \text { Value }}$ & Training sample & Testing sample & p Value \\
\hline Age, years, range & $48.2(41.5,57.4)$ & $48.1(41.0,57.6)$ & 0.59 & $48.8(41.7,58.7)$ & $48.5(41.3,58.9)$ & 0.43 \\
\hline WC, $\mathrm{cm}$ & $77.0(70.2,84.2)$ & $76.5(70.0,84.0)$ & 0.37 & $80.8(73.6,88.0)$ & $81.0(73.8,88.2)$ & 0.31 \\
\hline BMI, $\mathrm{kg} / \mathrm{m}^{2}$ & $23.4(21.2,26.0)$ & $23.3(21.2,25.8)$ & 0.35 & $23.4(21.1,25.7)$ & $23.3(21.1,25.8)$ & 0.64 \\
\hline WHR, unit & $0.82(0.75,0.87)$ & $0.82(0.78,0.87)$ & 0.12 & $0.87(0.82,0.91)$ & $0.87(0.83,0.92)$ & 0.20 \\
\hline Overweight, n (\%)* & $1156(31.5)$ & 1154 (31.4) & 0.95 & $1121(32.8)$ & $1083(32.2)$ & 0.55 \\
\hline Overall obesity, n (\%)† & $448(12.2)$ & $414(11.3)$ & 0.22 & $338(9.9)$ & $359(10.7)$ & 0.31 \\
\hline Central obesity, $\mathrm{n}(\%) \neq$ & $1460(39.8)$ & $1419(38.6)$ & 0.32 & $1261(37.0)$ & $1287(38.2)$ & 0.28 \\
\hline Type 2 diabetes, n (\%)§ & $258(7.3)$ & $243(6.6)$ & 0.48 & $241(7.1)$ & $252(7.5)$ & 0.50 \\
\hline
\end{tabular}

Continuous variables presented as median (25th percentile, 75th percentile) and categorical variables as $\mathrm{n}(\%)$.

${ }^{*}$ Diagnostic criteria for Chinese population: body mass index (BMI) $\geq 25 \mathrm{~kg} / \mathrm{m}^{2}$ and $\mathrm{BMl}<28 \mathrm{~kg} / \mathrm{m}^{2}$ was defined as overweight.

†Diagnostic criteria for Chinese population: $B M I \geq 28 \mathrm{~kg} / \mathrm{m}^{2}$ was defined as obesity.

¥Diagnostic criteria for Chinese population: waist circumference $(W C) \geq 85 \mathrm{~cm}$ for men or $W C \geq 80 \mathrm{~cm}$ for women was defined as obesity

$\S$ Percentage of diabetes in training/testing sample as number of diabetes/total number in training or testing sample.

WHR, waist-hip ratio. 


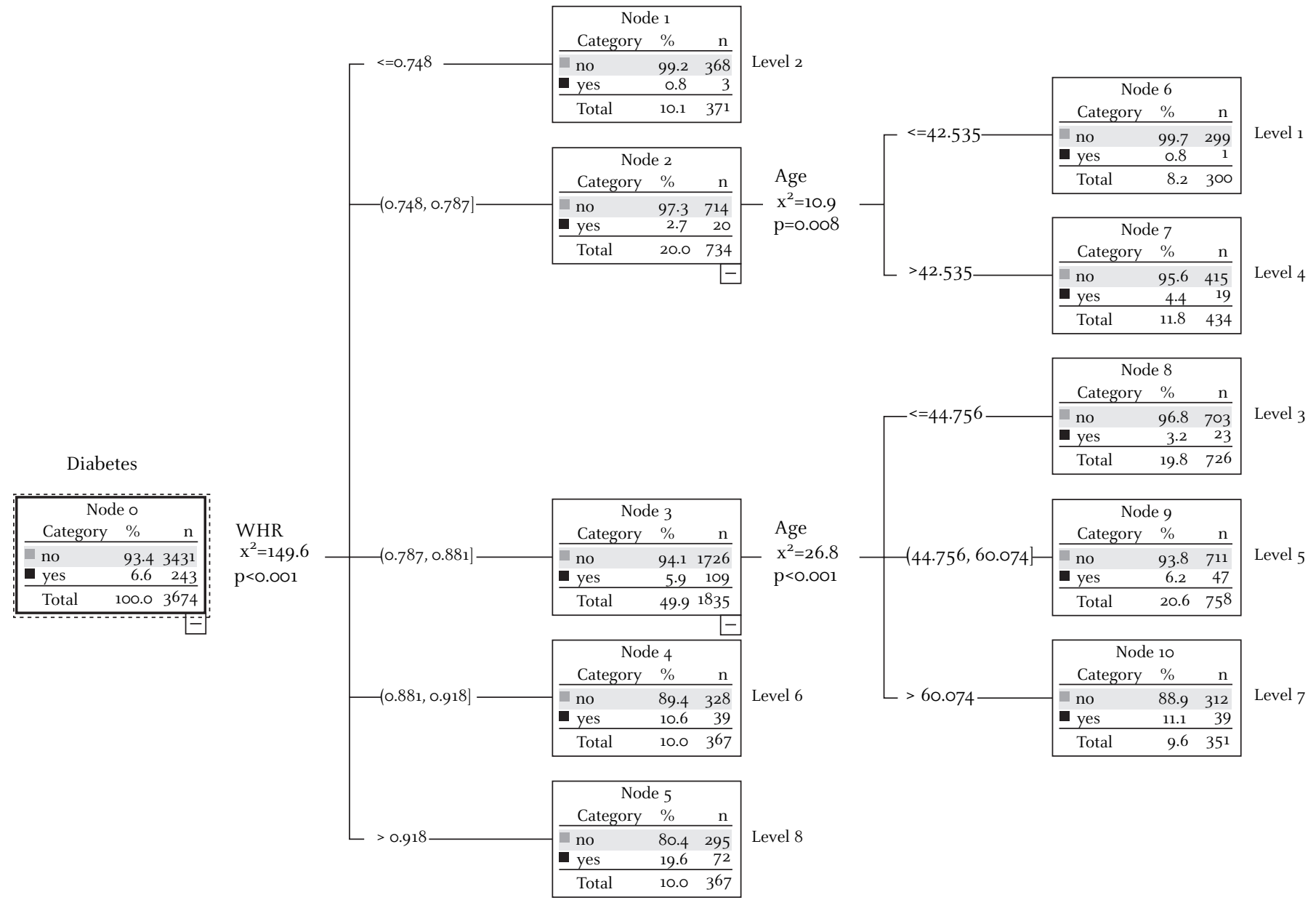

Figure 1 Predictors of type 2 diabetes and risk stratification by classification and regression tree analysis for women in the training sample. WHR, waist-hip ratio.

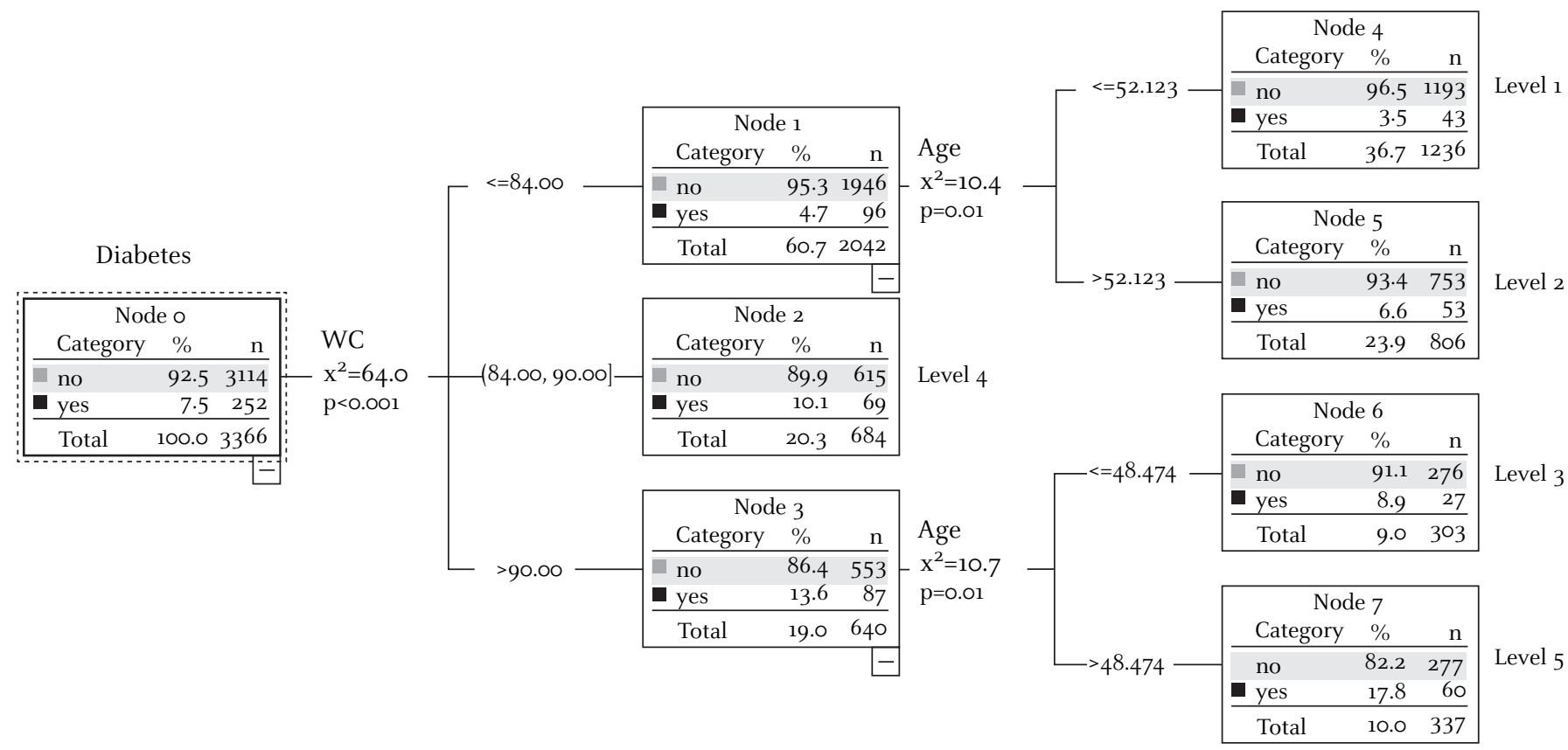

Figure 2 Predictors of type 2 diabetes and risk stratification by classification and regression tree analysis for men in the training sample. WC, waist circumference. 
Diabetes prevalence (\%) Women
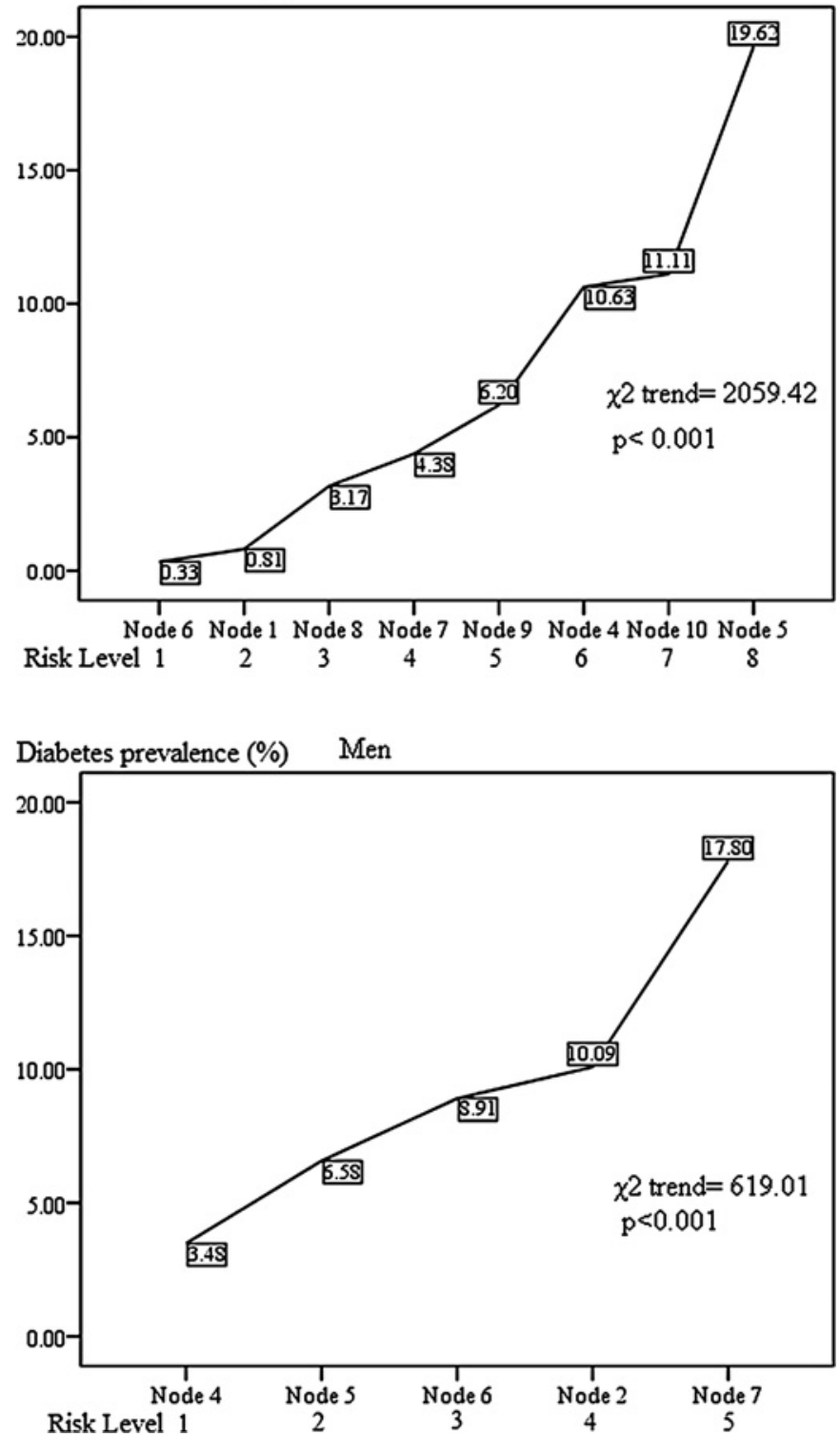

Figure 3 Type 2 diabetes prevalence rates of subgroups with different diabetic risk levels developed by classification and regression tree analysis in women and men.

in table 2. A threshold DRL of 6 or greater in women identified $62 \%$ of individuals with type 2 diabetes with a specificity of $72 \%$, and a threshold DRL of 3 or greater in men identified $60 \%$ of individuals with type 2 diabetes with a specificity of $63 \%$.

The CART analysis was able to stratify similar results in both training and testing samples (figures 1, 2, 4 and 5). The AUC in the training sample were not significantly different from those in the testing sample for both women and men (women $0.71 \mathrm{vs}$ 0.73 ; men 0.65 vs 0.69 ), indicating a good prediction above chance. Other diagnostic statistics were also similar in the two samples (table 2).

Multivariable (age, WHR, WC and BMI) logistic regression analyses show that age, WC and WHR were associated with type 2 diabetes in women $\left(p=\left(1+e^{-(0.048 \text { Age }+0.020 W C+4.01 W H R)}\right)^{-1}\right.$, and age, WC, WHR and BMI in men $\left(p=\left(1+e^{-(0.033}\right.\right.$ Age $+0.019 W C$ $+3.691 W H R+0.043 B M I))^{-1}$. There was no difference of AUC between CART analysis and the logistic regression model (data not shown).

\section{DISCUSSION}

In this study, a self-assessment tool with two simple predictors to identify individuals at high risk of type 2 diabetes was developed and successfully validated by CART analysis in a Chinese general population. The final child nodes generated by CART analysis stratified women into eight DRL and men into five DRL. Nearly two-thirds of individuals with type 2 diabetes have been identified with a reasonable specificity.

As a developing country, the cost on primary care has already been a colossal burden in China. Moreover, awareness, treatment and control of diabetes are relatively low among Chinese adults. $^{28}$ Therefore, it will be of great value to develop a simple tool for identifying individuals at high risk of type 2 diabetes. To the best of our knowledge, no screening tool for diabetes has been developed previously in the Chinese population. As the DRL generated by CART analysis in this study can be easily applied in the general population and can identify individuals with diabetes, there is great potential for its application in primary care settings in resource-limited countries.

The predictive performance and discriminative ability of this simple tool is highly comparable to those diabetes risk scores that are composed of different sets of clinical variables. ${ }^{11} 13141718$ The AUC of DRL generated by CART analysis in our study is similar to those in Indian (AUC 0.73), ${ }^{18}$ Thai (AUC 0.74) ${ }^{12}$ and Danish (AUC 0.76) ${ }^{14}$ studies. However, those diabetes risk scores included at least four variables and also required medical information. Even higher AUC (Germany 0.84; USA 0.85; UK 0.80; USA 0.82, Spain 0.83 for men, 0.87 for women; and middle east 0.83 ) have been reported if more risk factors are included, such as smoking, alcohol consumption and biochemical tests. ${ }^{11} 171923$ It is well known that the more variables included in the prediction tool, the less applicable it is to the general population. Given that diabetes could be prevented or delayed by changing lifestyle and physical activity patterns, it seems more sensible to target individuals at high risk by a simple programme that could be run easily in the general public.

The DRL in this study is very simple and also relatively easy to interpret. Compared with other diabetes risk scores, this DRL has a moderate sensitivity and a similar PPV (11-14\%), ${ }^{11} 1415$ but a high NPV (95-96\%). For identifying individuals with diabetes,

Table 2 Diagnostic statistics of the DRL for identifying type 2 diabetes in women and men

\begin{tabular}{|c|c|c|c|c|}
\hline \multirow{2}{*}{$\begin{array}{l}\text { Diagnostic statistics } \\
(95 \% \text { CI) }\end{array}$} & \multicolumn{2}{|l|}{ Women (DRL $\geq 6$ ) } & \multicolumn{2}{|l|}{ Men (DRL $\geq 3$ ) } \\
\hline & Training sample & Testing sample & Training sample & Testing sample \\
\hline Sensitivity & 0.61 (0.55 to 0.67$)$ & $0.62(0.55$ to 0.68$)$ & $0.59(0.52$ to 0.65$)$ & $0.59(0.52$ to 0.65$)$ \\
\hline Specificity & 0.71 (0.70 to 0.73 ) & $0.73(0.71$ to 0.74$)$ & $0.63(0.62$ to 0.65$)$ & 0.63 (0.61 to 0.64$)$ \\
\hline Negative predictive value & 0.96 (0.95 to 0.97$)$ & $0.96(0.96$ to 0.97$)$ & $0.95(0.94$ to 0.96$)$ & 0.95 (0.94 to 0.96$)$ \\
\hline Positive likelihood ratio & 2.16 (1.94 to 2.42 ) & 2.27 (2.02 to 2.54$)$ & 1.61 (1.43 to 1.80$)$ & $1.61(1.43$ to 1.80$)$ \\
\hline Negative likelihood ratio & $0.54(0.47$ to 0.64$)$ & $0.53(0.45$ to 0.62$)$ & $0.65(0.56$ to 0.76$)$ & 0.65 (0.55 to 0.76$)$ \\
\hline
\end{tabular}

$\mathrm{DRL}$, diabetes risk level. 


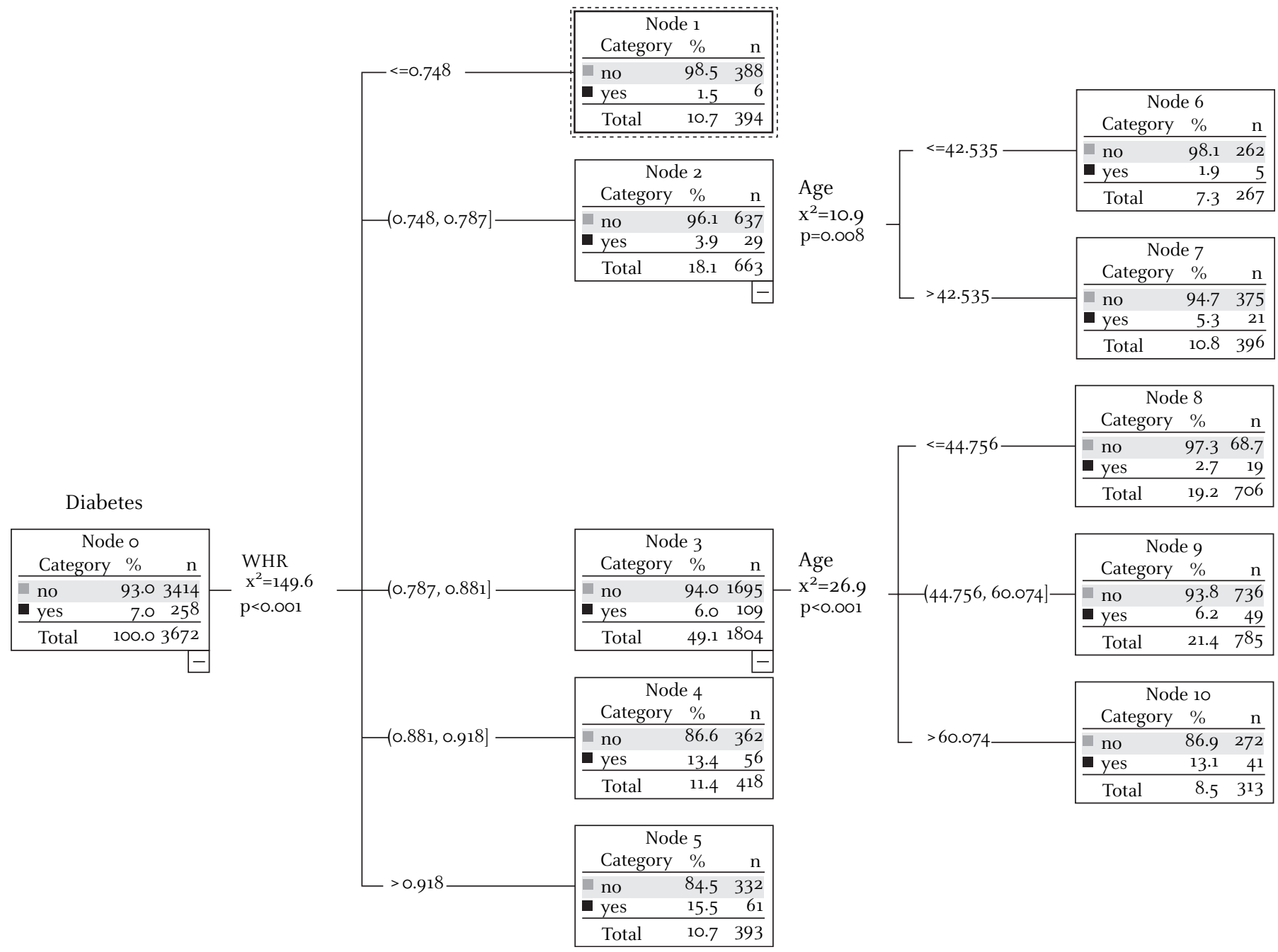

Figure 4 Predictors of type 2 diabetes and risk stratification by classification and regression tree analysis for women in the testing sample. WHR, waist-hip ratio.

the DRL has a sensitivity of $61 \%$ with a specificity of $71 \%$ in women and a sensitivity of $60 \%$ with a specificity of $63 \%$ in men. According to NPV, individuals with lower DRL are unlikely to have diabetes (approximately $95 \%$ probability). This DRL emphasises people with obesity, especially older people (women $>60$ years and men $>50$ years). Women with a DRL of 8 (WHR $>0.918$ ) and men with a DRL of 5 (WC $>84 \mathrm{~cm}$ ) will then need further blood tests and a suggestion to change to a healthier lifestyle for primary prevention. This information is very important for both the individuals and the health workers when making plans for action.

CART analysis has been proved to perform better than a logistic regression model in developing diabetes risk scores. ${ }^{11}$ In the current study, CART analysis provided similar results to multiple logistic regression models in terms of sensitivities, specificities and AUC. The results of CART analysis also confirmed that central adiposity (eg, WHR and WC) are better predictors of type 2 diabetes than overall obesity (BMI), which is consistent with previous studies. ${ }^{25}$

There are several advantages of the DRL. First, it is developed in a representative general Chinese population. Our study employed a multistage stratified random sampling method to select a representative national sample from the Chinese general population. Second, the development and validation of the model in two separate groups (randomised split sample) is an important strength, which lends support to the robustness and generalisability of the results. Third, the final model is quite simple, with only two items in a classification tree form. Compared with other diabetes risk scores derived from more variables and also including a blood test for glucose, ${ }^{11-14} 17$ the DRL can easily be applied at home in the general population. Although adding laboratory results would be more predictive, they were not available in the general public and, thus, were not included in our analysis. Our goal in this study is to develop a parsimonious tool, and not primarily to maximise prediction. Potential limitations of the current analysis must be addressed. First, the association between the independent variable and type 2 diabetes may not be causal given the cross-sectional nature of the current study. Second, our study did not include a younger age group.

\section{CONCLUSION}

The CART-based analysis in the current study has created a simple robust tool with considerable discriminative ability and is easy to apply in the general public. We strongly believe that the public health implications of this decision tree are considerable. It is a cost-efficient and practical tool to identify individuals at high risk of type 2 diabetes in the general population and also to offer a chance for early prevention measures. 


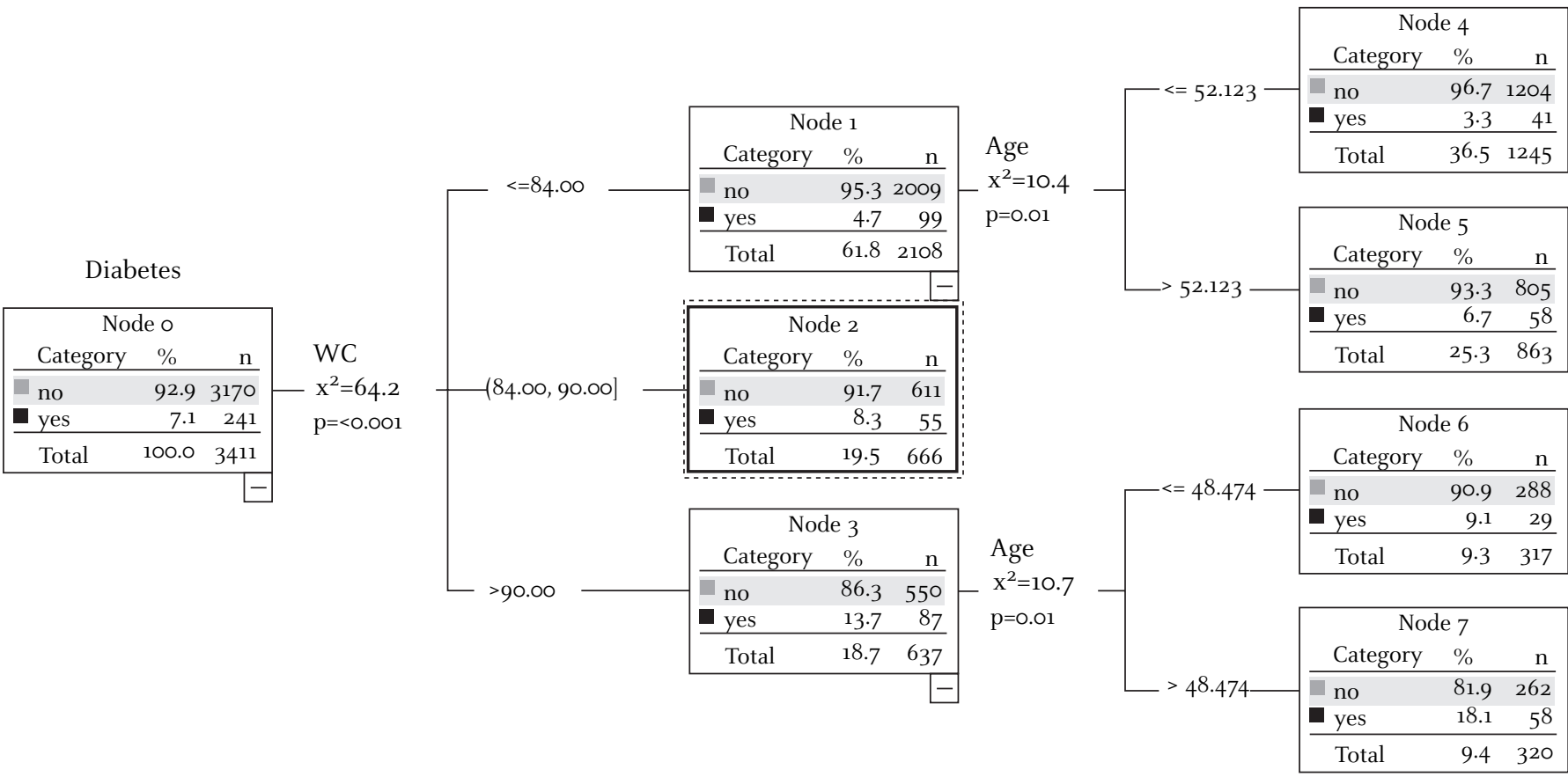

Figure 5 Predictors of type 2 diabetes and risk stratification by classification and regression tree analysis for men in the testing sample. WC, waist circumference.

\section{What is already known on this subject}

- Type 2 diabetes is associated with a number of genetic, environmental and lifestyle factors and could be prevented or delayed through simple lifestyle modification among high-risk individuals.

- Current available screening tools for identifying individuals at high risk of type 2 diabetes can be invasive, costly and time consuming.

- A screening tool for type 2 diabetes is lacking in the Chinese population.

\section{What this study adds}

- The present study used two easy self-assessment anthropometric measures (WHR and age for women and WC and age for men) to generate a new tool for identifying individuals at high risk of type 2 diabetes.

- This quick self-assessment tool has identified a substantial proportion of individuals with type 2 diabetes in the Chinese general population.

Acknowledgements The authors would like to thank Dr Cong Sun from the Centre for Eye Research Australia, University of Melbourne, for reading this paper.

Contributors All authors contributed to the paper. JX undertook the analysis and interpreted the results and drafted the paper. DH, DHY, JH, CSC and DFG critically revised the paper. All authors have seen and agreed the final draft. JX is guarantor for the paper.

Funding The InterASIA study was funded by a contractual agreement between Tulane University, Los Angeles, USA, and Pfizer Inc, New York, USA. Several researchers employed by Pfizer Inc were members of the study steering committee that designed the study. However, the study was conducted, analysed and interpreted by the investigators independently of the sponsor.
Competing Interests None.

Ethics approval The InterASIA study was approved by the Institutional Review Board at the Tulane University Health Sciences Center and the ethics committee and other relevant regulatory bodies in China.

Patient consent Obtained.

Provenance and peer review Not commissioned; externally peer reviewed.

\section{REFERENCES}

1. Wild S, Roglic G, Green A, et al. Global prevalence of diabetes: estimates for the year 2000 and projections for 2030. Diabetes Care 2004;27:1047-53.

2. Hancox RJ, Milne BJ, Poulton R. Association between child and adolescent television viewing and adult health: a longitudinal birth cohort study. Lancet 2004;364:257-62.

3. Sawada SS, Lee IM, Muto T, et al. Cardiorespiratory fitness and the incidence of type 2 diabetes: prospective study of Japanese men. Diabetes Care 2003:26:2918-22.

4. Das SK, Elbein SC. The genetic basis of type 2 diabetes. Cellscience 2006;2:100-31.

5. Gable D, Sanderson SC, Humphries SE. Genotypes, obesity and type 2 diabetes can genetic information motivate weight loss? A review. Clin Chem Lab Med 2007:45:301-8.

6. Weedon MN, McCarthy Ml, Hitman G, et al. Combining information from common type 2 diabetes risk polymorphisms improves disease prediction. PLoS Med 2006;3 e374.

7. Romao I, Roth J. Genetic and environmental interactions in obesity and type 2 diabetes. J Am Diet Assoc 2008:108(4 Suppl 1):S24-8.

8. Tuomilehto J, Lindstrom J, Eriksson JG, et al. Prevention of type 2 diabetes mellitus by changes in lifestyle among subjects with impaired glucose tolerance. N Engl J Med 2001:344:1343-50.

9. Liu J, Grundy SM, Wang W, et al. Ten-year risk of cardiovascular incidence related to diabetes, prediabetes, and the metabolic syndrome. Am Heart J 2007:153:552-8.

10. Jia WP, Pang C, Chen L, et al. Epidemiological characteristics of diabetes mellitus and impaired glucose regulation in a Chinese adult population: the Shanghai Diabetes Studies, a cross-sectional 3-year follow-up study in Shanghai urban communities. Diabetologia 2007:50:286-92.

11. Heikes KE, Eddy DM, Arondekar B, et al. Diabetes risk c: a simple tool for detecting undiagnosed diabetes and pre-diabetes. Diabetes Care 2008;31:1040-5.

12. Aekplakorn W, Bunnag $\mathrm{P}$, Woodward $\mathrm{M}$, et al. A risk score for predicting incident diabetes in the Thai population. Diabetes Care 2006;29:1872-7.

13. Stern MP, Williams K, Haffner SM. Identification of persons at high risk for type 2 diabetes mellitus: do we need the oral glucose tolerance test? Ann Intern Med 2002; 136:575-81.

14. Glumer C, Carstensen B, Sandbaek A, et al. A Danish diabetes risk score for targeted screening: the Inter99 study. Diabetes Care 2004;27:727-33. 
15. Lindstrom J, Tuomilehto J. The diabetes risk score: a practical tool to predict type 2 diabetes risk. Diabetes Care 2003;26:725-31.

16. Schmidt MI, Duncan BB, Bang $\mathrm{H}$, et al. Identifying individuals at high risk for diabetes: the atherosclerosis risk in communities study. Diabetes Care 2005;28:2013-18.

17. Cabrera de LA, Coello SD, Rodriguez Perez MC, et al. A simple clinical score for type 2 diabetes mellitus screening in the Canary islands. Diabetes Res Clin Pract 2008;80:128-33.

18. Vivek Chaturvedi KSR, Prabhakaran D, Jeemon P, et al. Development of a clinical risk score in predicting undiagnosed diabetes in urban Asian Indian adults: a population-based study. CVD Prevention and Control 2008:3:141.

19. Griffin SJ, Little PS, Hales CN, et al. Diabetes risk score: towards earlier detection of type 2 diabetes in general practice. Diabetes Metab Res Rev 2000;16:164-71.

20. Glumer C, Vistisen D, Borch-Johnsen K, et al. Risk scores for type 2 diabetes can be applied in some populations but not all. Diabetes Care 2006;29:410-14.

21. Ramachandran A, Snehalatha C, Vijay V, et al. Derivation and validation of diabetes risk score for urban Asian Indians. Diabetes Res Clin Pract 2005;70:63-70.

22. Mohan V, Deepa R, Deepa $\mathrm{M}$, et al. A simplified Indian diabetes risk score for screening for undiagnosed diabetic subjects. J Assoc Physicians India 2005;53:759-63.

23. Al-Lawati JA, Tuomilehto J. Diabetes risk score in Oman: a tool to identify prevalent type 2 diabetes among Arabs of the Middle East. Diabetes Res Clin Pract 2007:77:438-44.
24. Liu Y, Berman P, Yip W, et al. Health care in China: the role of non-government providers. Health Policy 2006;77:212-20.

25. Hu D, Xie J, Fu P, et al. Central rather than overall obesity is related to diabetes in the Chinese population: the InterASIA study. Obesity 2007:15:2809-16.

26. Gu D, Reynolds K, Duan X, et al. Prevalence of diabetes and impaired fasting glucose in the Chinese adult population: International Collaborative Study of Cardiovascular Disease in Asia (InterASIA). Diabetologia 2003;46:1190-8.

27. Muntner $\mathbf{P}$, Gu D, Wildman RP, et al. Prevalence of physical activity among Chinese adults: results from the International Collaborative Study of Cardiovascular Disease in Asia. Am J Public Health 2005;95:1631-6.

28. Hu D, Fu P, Xie J, et al. Increasing prevalence and low awareness, treatment and control of diabetes mellitus among Chinese adults: the InterASIA study. Diabetes Res Clin Pract 2008;81:250-7.

29. The Expert Committee on the Diagnosis and Classification of Diabetes Mellitus. Report of the expert committee on the diagnosis and classification of diabetes mellitus. Diabetes Care 1997;20:1183-97.

30. Zhou B. Prospective study for cut-off points of body mass index in Chinese adults. Zhonghua Liu Xing Bing Xue Za Zhi 2002;23:431-4.

31. Lemon SC, Roy J, Clark MA, et al. Classification and regression tree analysis in public health: methodological review and comparison with logistic regression. Ann Behav Med 2003;26:172-81.

32. Steinberg D, Colla P. CART: tree-structured non-parametric data analysis. San Diego CA: Salford Systems, 1995. 